\title{
Tycho's illusion and human cognition
}

Sir - For several decades at the start of the seventeenth century, the main cosmological alternative to the Sun-centred Copernican system was not the Ptolemaic, but a compromise system proposed by Tycho Brahe (Fig. 1). In the Tychonic system, the planets move around the Sun exactly as Copernicus proposed. But the Sun, accompanied by the orbits of the planets, moves around the Earth. This new system appeared to embrace the elegance of Copernicus yet avoid his heresies. But in this system the orbit of the Sun and the orbit of Mars intersect, so it seemed obvious both in Tycho's time and our own that the system is inconsistent with the traditional view that the Sun and planets are carried by solid spheres.

In fact, the collision seen in the Tychonic diagram is an illusion, which has deceived the experts for 400 years. From Kepler in Tycho's time to Thomas Kuhn and many others in our own, there has been no dissent. Why?

Figure 1 separates Tycho's original diagram into two components: the cut-out, Fig. 1a, contains everything that shares in the Sun's annual motion and nothing else; Fig. $1 \mathrm{~b}$ is what is left of Tycho's diagram after removing the cut-out. To run the model, make an enlarged photocopy of the figures, cut the two apart, and then fold the cut-out to bisect the black circular region in its centre. Next, cut the black centre out and trim away the remaining black on the periphery. Then unfold the cut-out and centre its hole over the Earth-Moon region of the template. Turning the cut-out will produce the Tychonic orbit of the Sun, accompanied (as Tycho and observations require) by the orbits of the planets.

On a scale of about 1 to $10^{12}$, the cut-out is a model of a single Tychonic sphere that carries six epicycles (the five planetary orbits plus a solar epicycle too small to show). This replaces Ptolemy's six spheres,
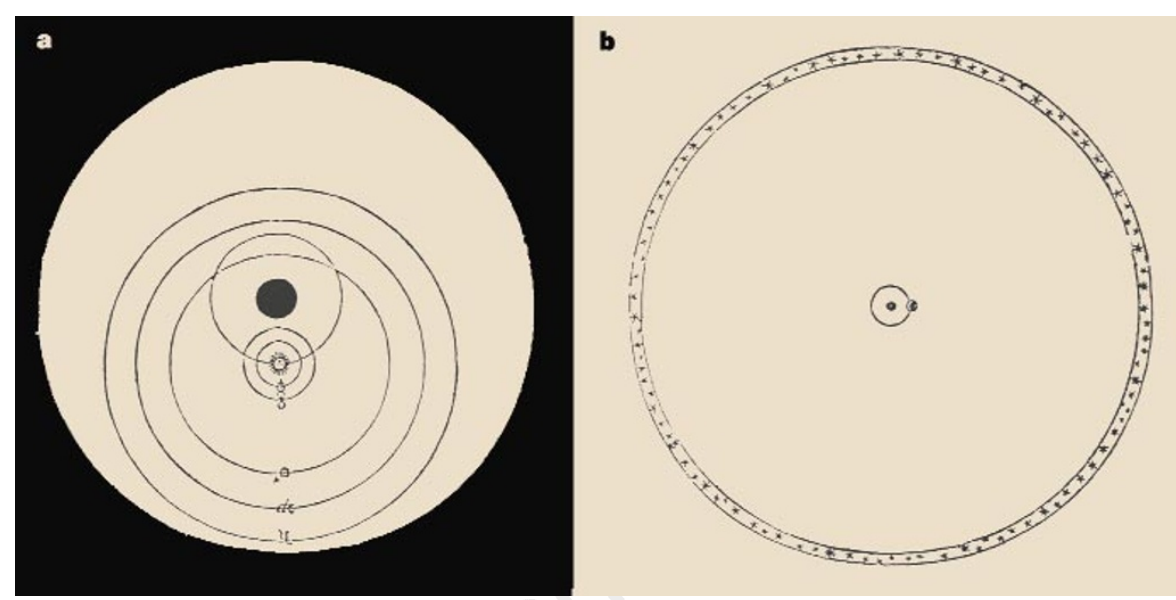

Figure 1 The Tychonic sphere in two parts. See text for instructions.

each carrying one epicycle. The single Tychonic sphere carries both the Sun and its tributary orbits in the common annual motion that the logic of the system requires. Within that single sphere, the heliocentric orbits would be physical objects carrying the planets. But the path of the Sun in Tycho's diagram does not carry anything Mars could no more collide with that mathematical locus than a ship could collide with the equator. But an expert in this matter, even more than a non-expert, usually cannot believe that until actually seeing the cut-out move. Then the illusion of an unavoidable collision is destroyed, and it becomes hard to believe that for 400 years no one questioned the collision.

What makes the illusion? Our experience is overwhelmingly that little things circle big things. But with Tycho's system the large orbits of the outer planets must swing around the Earth on the smaller radius shared with the Sun. If a person tries to imagine that rotation, intuition about how things move rebels. In the mind's eye, the Sun goes around the Earth accompanied by the smaller orbits of Venus and Mercury (as it should), but the larger orbits stay put, which they should not. This discrepancy cannot be conscious, for a person would then recognize it as logically absurd. But with the cut-out, it is easy to see that the orbits are carried by rotation of the entire region between the Earth/Moon system and the fixed stars. Now we see big carrying small, which feels intuitively comfortable, and the illusion disappears.

There has been much debate among psychologists about whether cognitive illusions can provide real insight into human reasoning, or reveal only how clever experimenters can trick naive subjects. But Tycho's illusion is plainly no mere laboratory trick. It has been deluding the experts for 400 years, and on a question they have taken very seriously.

A full paper discussing the illusion and its cognitive implications is available at http://www.harrisschool.uchicago.edu/ Tycho.html.

Howard Margolis

Harris School,

University of Chicago,

1155 E 60th Street,

Chicago, Illinois 60637, USA

e-mail:hmarg@uchicago.edu

\section{Art in the round}

Sir - How lovely to see the word "bollocks" appearing, perhaps for the first time, in Nature $(392,663 ; 1998)$. And how appropriate that it should occur in Martin Kemp's series of pieces exploring the assimilation of scientific ideas into artistic creation.

It has been said that scientists tend to have a greater appreciation of art than artists have of science. Kemp's illustrations, together with his report of the unnamed biology graduate student's thoughtful appraisal of Cornelia Parker's work, provide a powerful counter-argument to this assertion.

A fellow-scientist of my acquaintance recently ridiculed a monochrome painting at an exhibition into which she had presumably stumbled by mistake, or to escape from bad weather; superficially 'simple' modern works - Mark Rothko's canvases and Carl Andre's sculptures come to mind - are especially vulnerable. I suggested to my colleague that an article in Nature might be no more intelligible to the painter than this painting was to her, but that the artist would be unlikely to dismiss the article as casually as she had the painting.

If the University of Leicester student is working towards a $\mathrm{PhD}$, and if it is not too late, I would urge the student's supervisor to invite Cornelia Parker to act as an external examiner for the thesis. Ms Parker can hardly be any less eligible to evaluate this student's work than the student is to assess hers.

She could announce her verdict in the departmental tea room.

Ian Smith

18 Lockwood Street,

York YO31 7QY, UK

e-mail:i.smith@csl.gov.uk 